\title{
Effects of a Music-Movement Program in Elementary School Physical Education Classes on Pupils' Motor Skills
}

\author{
Dimitrios Kyriazis $^{1^{*}} \quad$ Eva Pavlidou$^{1} \quad$ Vassilis Barkoukis $^{2} \quad$ Eleni Fotiadou ${ }^{2}$ \\ 1.School of Early Childhood Education - Aristotle University of Thessaloniki, Education Faculty Tower \\ Thessaloniki 541 24, Greece \\ 2.School of Physical Education and Sports Science- Aristotle University of Thessaloniki, 57001 Thermi
}

Thessaloniki, Greece

\begin{abstract}
This study aimed to develop a short music-movement program and evaluate its effectiveness in improving elementary school pupils' motor skills. Two hundred forty-five pupils (10.5 \pm .526 years) randomly assigned into intervention and control group participated in the study. The pupils performed two tasks, selected from the Bruinninks Oseretsky Motor Proficiency Test (2nd edition) related to rhythmic coordination: (a) Dribbling a ballAlternating hands and (b) Stepping sideways over a balance beam. All Participants completed the tests before, immediately after and two months after the implementation of the program. Pupils in the intervention group attended the music-movement program during the warm-up phase of the standard Physical Education (PE) lesson, whereas those in the control group attended the regular PE warming up according to the national curriculum. The program was implemented for 13 weeks twice a week. The results of the ANOVA with repeated measures on time revealed a significant improvement of both motor skills in intervention group pupils as compared to control group pupils. A follow-up measurement indicated that the effect of the program was maintained two months after the intervention. The findings of the study support the effectiveness of a music-movement program during the warmup phase of the PE lesson on fifth and sixth grade pupils' motor skills.
\end{abstract}

Keywords: motor skills, physical education, music-movement, intervention program, warm-up phase, rhythmic coordination

DOI: $10.7176 / \mathrm{JEP} / 10-21-11$

Publication date:July $31^{\text {st }} 2019$

\section{Introduction}

Motion is based on rhythm and therefore it helps children perform fundamental movements with dexterity, and in turn supports motor development (Galahue, 1996). The combination of movement and music provides the education process with the opportunity to bring the children in contact with basic elements of music, (rhythm, melody and singing) in a pleasant and creative way (Campbell, 2000). Hotz (1992) and Erikson (2004) suggested that rhythm assists in motor learning, through the pleasant mood established in the presence of music. When motions are accompanied by music and rhythm, they can become meaningful and performed more easily and efficiently (Karageorgis \& Terry, 1997; Sousou, 1997) and with increased precision (Martin \& Ellerman, 2001). Music movement programs help children not only to discover the movement's rhythmic structure but also to improve the quality of their movement and coordination skills and their school achievement (Pavlidou, 1998). Moreover, rhythmic movement programs are perceived as energetic and enjoyable activities (Zachopoulou, Derri, Chatzopoulos, \& Ellinoudis, 2003) that improve the pupils' concentration (Juntunen, Perkiö, \& Simola-Isaksson, 2010; Fross, 2000) and improve the development of skills which are essential for future evolution in sport (Pica, 1999).

Prior studies implementing music-movement programs were largely focused on preschool education and supported the effectiveness of these programs on preschoolers' motor development (Brown, Sherill \& Gench 1981; Deli, Bakle \& Zachopoulou, 2006; Venetsanou \& Kambas 2004; Zachopoulou, Tsapakidou \& Derri, 2004) and the rhythmic ability (Bailey \& Penhune, 2010; High, 1994; Moor, 1984; Pollatou et al., 2012; Venetsanou, Dondi, \& Koutsouba, 2014; Zachopoulou et al., 2003; Weikart, 1989), and cognitive development of preschool children (Blasi \& Foley, 2006; Derri, Tsapakidou, Zachopoulou, \& Gini, 2001; Freshwater, Sherwood, \& Mbugua, 2008; Gillespie \& Glider, 2010; Salmon, 2010; Winsler, Ducenne, \& Koury, 2011). Moreover, Tsapakidou, Zachopoulou and Zografou (2001) combined music movement methods proposed by Orff (Keetman, 1974) and Dalcroze (Bachman, 1991), and found that their implementation for a three months period improved the spatial perception on 4-6 age pupils', their reaction to audio instructions, their rhythmic ability and their ability of taking decisions. Still, the number of studies investigating the effect of music-movement programs on elementary and secondary school children is rather limited. Notable exceptions are the studies by Likesas and Zachopoulou (2006), Lykesas, Koutsoumba and Tyrovola (2009) and Chatzipandeli, Pollatou, Diggelidis and Kourtesis (2007). These studies also support the effectiveness of music movement programs' on motor skills of pupils' attending fifth and sixth grade. Specifically, Likesas and Zachopoulou (2006) have indicated that a musical movement program increases elementary pupils' intrinsic motivation and pleasure while teaching Greek traditional dances during physical 
education lessons. Similarly, Chatzipandeli et al. (2007) demonstrated that a program including exercises accompanied by music, improved 1st-grade elementary pupils' manipulative skills. Moreover Lykesas et al. (2009) reported a strong effect on secondary pupils' creativity during lessons teaching traditional dance. In addition, Murray (1975) recommends teachers to use dance in elementary education because of its effectiveness on rhythmic abilities and many other motor and non-motor abilities. Moreover, Kyriazis, Pavlidou, Barkoukis \& Fotiadou, (2017) supported that a short music movement program implemented in the warm-up phase of the physical education lesson for 13 weeks can significantly improve 5th and 6th-grade elementary school pupils' rhythmic ability in handclapping and walking in both simple $(2 / 4)$ and complex $(7 / 8,9 / 8)$ rhythmic patterns. This evidence demonstrates that in order to promote pupils' motor abilities, rhythmic exercises can effectively be applied in higher grades in elementary school too. This is consistent with view that motor ability development is related to the central nervous system (CNS) and, thus, is strongly related to maturation and activation of motor control mechanisms (Cratty, 1975). According to past evidence human motor development and cognitive functions are intertwined (Haapala et al., 2013). However, such activities are typically integrated only in the first three grades of elementary school.

Furthermore, past evidence demonstrated that rhythms with a regular temporal structure are distinguished and reproduced better than irregular rhythms (Grahn \& Brett, 2007; Patel, Iversen, Chen, \& Repp, 2005). Moreover, simple rhythms (i.e., $2 / 4,6 / 8$ ) can be used for younger pupils aged between 6 and 8 years-old, whereas more complex rhythms (i.e., 7/8 and 9/8) can be used in older elementary school pupils (Serbezis \& Panagopoulou, 2008). Such rhythms have been found to effectively increase pupil's rhythmic ability (Kyriazis et al., 2017; Pavlidou, Mertzanidou, \& Zissi, 2009). Importantly, as Zachopoulou et al. (2003) noted these activities are enjoyable and do not require special equipment.

Typically, according to the national physical education curricula the time allocated in music-movement education is decreased in higher grades of the elementary schools in favour of games and sports (Hardman, Klein, Patriksson, Rychtecký, \& da Costa, 2008). However, considering that music-movement education in these grades can still help pupils increase their motor development and better learn sport skills, music movement education should be integrated in the physical education lesson. To manage this without interrupting the teaching of the other physical education subjects, but assisting them, a possible way could be to integrate music movement drills during the warm-up phase of the lesson. This approach is expected to have two benefits for the lesson, a) providing an alternative, enjoyable and interesting way to initiate the lesson and b) maintaining teaching rhythmic motor skills to pupils. Past evidence showed that music-movement programs are perceived as energetic and enjoyable activities (Zachopoulou et al., 2003) and include body activities in a low or medium intensity of aerobic exercise that can warm up pupils for a physical education lesson (Faigenbaum, Bellucci, Bernieri, Bakker, \& Hoorens, 2005). However, so far there is only limited evidence that such an approach could be implemented in the school setting and whether it cans effectively increasing pupils' motor skills. Therefore, the aim of the present study was to develop a music-movement program suitable for the warm up phase of the physical education lesson and test its effectiveness in improving elementary school pupils' selected motor skills related to rhythmic coordination. Based on the abovementioned literature it was hypothesised that a short music-movement program in the warm up phase of the lesson will improve elementary school pupils' skills on (a) dribbling a ball - Alternating hands and (b) stepping sideways over a balance beam.

\section{Method}

2.1 Sample and Procedure

A convenient sample from schools accepting to take part in the study was used. Overall, seven schools participated in the study randomly allocated into intervention (three schools) and control (four schools) group. The sample consisted of 245 elementary school pupils (122 boys, 123 girls) with an age range between 10 to 12 years-old (M $=10.5, \mathrm{SD}=.52$ ). Parents provided consent form allowing their children to participate in the study. Students were attending typical co-educational elementary schools in a large city of Northern Greece and were assigned into groups of intervention ( $\mathrm{n}=123$ pupils) and control ( $\mathrm{n}=122$ pupils).

Each participant was asked to perform the two selected motor items of the BOT2 related to rhythmic coordination: (a) Dribbling a ball-Alternating hands and (b) Stepping sideways over a balance beam for 15 seconds. The first test required pupils to dribble a tennis ball with alternating hands aiming to the highest number of achieved dribbles. An examiner was counting the successful dribbles and stopped if the subject lost the ball, or was dribbling with the same hand. In the second test pupils had to step sideways over a $8 \mathrm{~cm}$ high balance beam in $15 \mathrm{sec}$. The examiner counted the number of steps within an interval of $15 \mathrm{sec}$ (using a stopwatch), which was considered as correct only if both feet touched the ground simultaneously on the same side of the beam. The pupils were properly informed about the context of the test and were allowed some attempts without being recorded. In the beginning of the test, a set of four warning sounds accompanied the verbal instruction of the examiner "one, two, three, go", to start the corresponding test.

Three measurements were performed. A baseline pre-test measurement before the implementation of the 
intervention, a post-test measurement immediately after the intervention, and a follow up test two months after the post-test measurement. In the follow-up test, the $30 \%$ of the initial sample participated (33 pupils in the intervention group and 33 in the control group).

\subsection{Bruininks-Oseretsky Test of Motor Proficiency (BOT-2)}

The Bruininks-Oseretsky Test of Motor Proficiency, 2nd Edition (BOT-2; Bruininks \& Bruininks, 2006) is a pack of tests with the purpose of providing a comprehensive overview of fine and gross motor skills in children and youth, within 4 and 21 years. The BOT-2 has a Complete and a Short Form and is intended for practitioners and researchers to characterize motor performance, like fine manual control, manual coordination, body coordination, strength and agility. The entire test requires 45 to 60 minutes for administration; however, separate composite areas can be administered in 10 to 15 minutes (Deitz, Kartin, and Kopp (2007) investigated its validity and reliability which was high in all forms $(\mathrm{r}>.90)$. Two items, related to rhythmic coordination, were selected in the present study to examine the effectiveness of the intervention music-movement program on pupils' motor skills. The first test was the 6th item of the category "Upper limp coordination" in which pupils were required to dribble a tennis ball with alternating hands aiming to the highest number of achieved dribbles. The second test was the 2nd item of the category "Running-Speed and Agility" of the BOT-2 in that pupils had to step sideways over an $8 \mathrm{~cm}$ balance beam in $15 \mathrm{sec}$ (Figure 1 ).
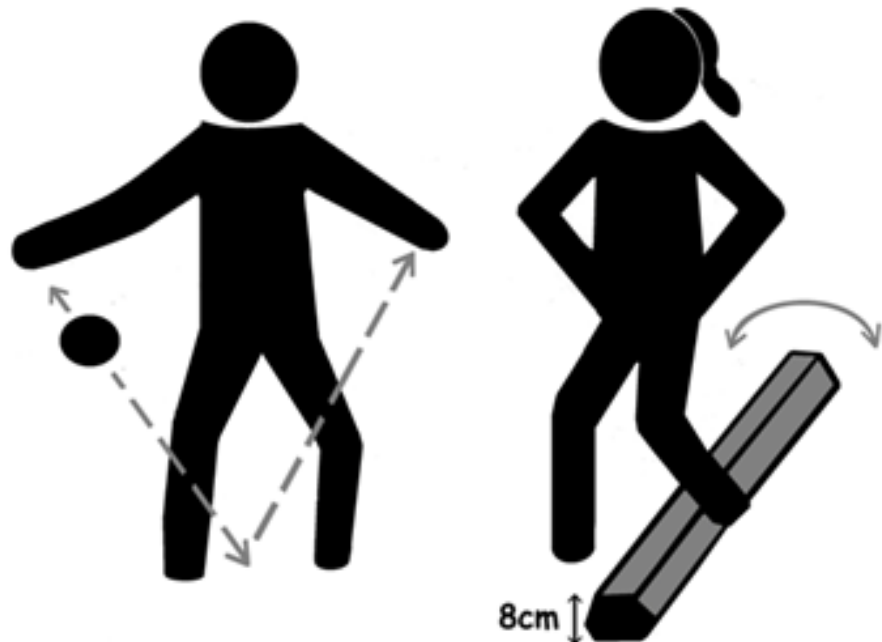

Figure 1. The two selected motor tests of BOT2

\subsection{The Intervention Program}

Taking into account the features of a regular physical education lesson, the intervention program was designed to last as long as its warm-up phase, i.e., 8 to $10 \mathrm{~min}$. Pupils in the intervention group performed the program twice a week for 13 weeks without interruption. Four independent thematic activities including motor activities accompanied by traditional and modern music constituted the program. The thematic activities included: 1) The motorcycle rider: The pupils impersonated motorcycle travellers who make a trip, meeting different cultures and various incidents in the towns they come through; 2) Old Greek cinematography: the scenario was connected to popular movie themes of old Greek cinematography; 3) World Music: pupils impersonating national dancers with corresponding rhythms travelled around the world; and 4) The Rhythm of Sports: pupils impersonated sports activities with suitable accompanying music. Each thematic activity was designed in an audio file which included the music and incorporated instructions according to the corresponding scenario in a narrative-storytelling form. Storytelling is the method that supports children's development, to help them express and assign meaning to the world, to develop communication, recognition, and recall skills. From elementary to high school, storytelling activities are proposed to students in order to improve their linguistic and literacy skills, and to foster their ability of interpretation, analysis, and synthesis (Garzotto, Paolini \& Sabiescu, 2010). Connections with the storyteller could create students' personal links with the stories and connect them with their own lives. In this way teacher educators believed that the stories encourage the development of empathy through emotional and embodied reactions to the stories (Garret, 2006) and motivate students more actively engage with the lesson (Davidson, 2004). The above elements had been taken into account to insert storytelling in the present intervention program helping pupils to be linked with the corresponding thematic activity.

During the activities, pupils intended to synchronize their motions (running, jumping, clapping, etc.) with the changes of the mixed rhythmic patterns $(2 / 4,3 / 4,7 / 8,9 / 8)$ and tempos (andante, moderato, allegro, presto, etc). These music themes and rhythmic patterns were chosen as the more appropriate for this age group. A combination 
of traditional and modern music was selected in order to create an enjoyable atmosphere with several rhythmic and tempo alternations. Also, this variety of rhythms facilitated the application of many different motor activities, relevant to the corresponding beat and providing the opportunity for expression in the thematic scenario.

Each scenario lasted 9-10 min, including a 1-1.5 min stretching period with relaxing music and only one of the four activities was applied each time. The activities were performed after starting the audio file of the corresponding scenario via audio player in the introduction phase of the physical education lesson (warming up). The physical education teacher participated in the program acting as a model providing additional guidance. Thus the acoustic scenario with music called the pupils to dance, run, jump and clap etc following the physical educators' instructions. Pupils in the control group performed the regular warming up according to the national curriculum. This warming up includes running and stretching exercises or a running-related game and stretching exercises for 8 to $10 \mathrm{~min}$. Students in the control group attended the typical PE program including teaching motor skills of common team and individual sports.

\subsection{Data Analysis}

SPSS 22.0 was used to test the study's hypotheses. Descriptive analyses were also performed. The study's hypotheses were tested via analyses of variance with repeated measures on time. The level of significance was set at $\mathrm{p}=.05$.

\subsection{Results}

Fourteen participants were excluded from the analysis as they didn't provide complete data in the pre- and posttest measurements. Therefore, the data from 109 pupils attending the intervention group and 122 pupils in the control group were used in the analyses. Participants in both groups did not differ in demographics in the baseline measurement. The mean values and standard deviations obtained by the two tests are summarized in Table 1 .

Table 1. Mean values and the standard deviation of the examined motor tests

\begin{tabular}{|c|c|c|c|c|c|c|c|c|c|c|c|c|}
\hline & \multicolumn{4}{|c|}{ Pre } & \multicolumn{4}{|c|}{ Post } & \multicolumn{4}{|c|}{ Follow Up } \\
\hline & \multicolumn{2}{|c|}{$\begin{array}{c}\text { Intervention } \\
\text { Group } \\
(\mathbf{n}=109)\end{array}$} & \multicolumn{2}{|c|}{$\begin{array}{l}\text { Control } \\
\text { Group } \\
(n=122)\end{array}$} & \multicolumn{2}{|c|}{$\begin{array}{c}\text { Intervention } \\
\text { Group } \\
(\mathbf{n}=109)\end{array}$} & \multicolumn{2}{|c|}{$\begin{array}{l}\text { Control } \\
\text { Group } \\
(n=122)\end{array}$} & \multicolumn{2}{|c|}{$\begin{array}{c}\text { Intervention } \\
\text { Group } \\
(\mathrm{n}=109)\end{array}$} & \multicolumn{2}{|c|}{$\begin{array}{l}\text { Control } \\
\text { Group } \\
(n=122)\end{array}$} \\
\hline & Mean & SD & Mean & SD & Mean & SD & Mean & SD & Mean & SD & Mean & SD \\
\hline $\begin{array}{l}\text { Dribbling a ball- } \\
\text { Alternating hands }\end{array}$ & 21.48 & 17.619 & 21.23 & 13.780 & 31.39 & 19.756 & 20.02 & 12.122 & 28.33 & 12.903 & 20.73 & 8.854 \\
\hline $\begin{array}{l}\text { Stepping sideways } \\
\text { over a balance beam }\end{array}$ & 20.91 & 3.655 & 20.04 & 3.024 & 24.04 & 2.738 & 20.16 & 2.977 & 23.58 & 2.750 & 20.24 & 2.424 \\
\hline
\end{tabular}

Two analyses of variance with repeated measures on time between pre-post and post-follow up measurements revealed significant group by time interactions in the examined motor tests (Table 2). Specifically, the pre-post analyses demonstrated a statistically significant increase in the performance of pupils in the intervention group in "Dribbling a ball-Alternating hands" $\left(\mathrm{F}=68.321^{* *}, \eta 2=.231, \mathrm{p}<.01\right)$ and in "stepping sideways over a balance beam" $\left(\mathrm{F}=100.467^{* *}, \eta 2=.306, \mathrm{p}<.01\right)$, compared to the pupils in the control group whom scores remained rather stable. Respectively the post-fu interaction analyses demonstrated that the effect of the intervention remained significant in the follow-up measurement two months later in "Dribbling a ball-Alternating hands" $\left(\mathrm{F}=3.253^{* *}\right.$, $\eta 2=.049, \mathrm{p}<.01)$ and in "stepping sideways over a balance beam" $\left(\mathrm{F}=3.239^{* *}, \eta 2=.049, \mathrm{p}<.01\right)$. The interactions values between measurements and groups obtained by the two tests are summarized in Table 2 . 
Table 2. Interactions between measurements and groups

\begin{tabular}{lcccc}
\hline & \multicolumn{1}{c}{$\begin{array}{c}\text { Between groups pre and post } \\
\text { measurements } \\
\text { Interaction }\end{array}$} & $\begin{array}{c}\text { Between groups post and fu } \\
\text { measurements } \\
\text { Interaction }\end{array}$ \\
\hline Dribbling a ball-Alternative hands & $68.321^{* *}$ & .231 & 3.253 & $\eta^{2}$ \\
Stepping sideways over a balance beam & $100.467^{* *}$ & .306 & 3.239 & .049 \\
\hline${ }^{*} p<.05^{* *} p<.01$. & & & &
\end{tabular}

\section{Discussion}

The aim of this study was to examine the effect of a music movement intervention program during the warm-up phase of the physical education lesson on the motor skills related to rhythmic coordination of elementary school pupils. The results of the analyses indicated that a music movement program used in the warm-up phase was effective in improving pupils' motor skills as compared to the regular warming up.

These findings are in line with those reported in previous studies with pre-schoolers demonstrating that such music movement intervention programs can positively influence motor and cognitive development (Blasi \& Foley, 2006; Derri et al., 2001; Freshwater et al., 2008; Gillespie \& Glider, 2010; Salmon, 2010; Winsler et al., 2009; Zachopoulou et al., 2004) and rhythmic ability (Bailey \& Penhune, 2010; High, 1994; Moor, 1984; Pollatou et al., 2012; Venetsanou et al., 2014; Zachopoulou etal., 2003). Additionally, they support previous evidence with elementary school pupils demonstrating that a music movement program can effectively improve various abilities and skills in older elementary and secondary school pupils too (Chatzipandeli et al., 2007; Kyriazis et al., 2017; Likesas \& Zachopoulou, 2006; Lykesas et al., 2009).

Past evidence has largely relied on improving motor skills and rhythmic ability implementing musicmovement programs on pre-schoolers neglecting elementary school pupils. Importantly, the results of the present study indicate that music movement programs are similarly effective in increasing motor skills related to rhythmic coordination of fifth and sixth grade elementary school pupils as well. It is also important to note that this increase was achieved by incorporating rhythmic activities in the lesson's warm-up phase. This has important implications for physical education lesson as it implies that a short period of implementation is adequate to maintain and improve the motor skills of 10 to 12 years old pupils. Physical education teachers can assist in the multifaceted motor development of their pupils incorporating such activities in the warm-up phase without costs on teaching other subjects (i.e., games etc). Interestingly, past evidence has shown that such rhythmic activities increase enjoyment from the lesson (Zachopoulou et al., 2003). Similarly in the present study, the participant pupils of the intervention music movement program were motivated by the pleasant atmosphere and acted vividly. In addition, the program's design in a storytelling form adding a lot of interpretation elements is thought to have contributed significantly to its effectiveness due to the pupils' active involvement (Davidson, 2004). These were the most important elements that resulted in the success of the program, as pupils were positively inspired and motivated to participate without compulsion, which is usually the case in activities they don't like. Therefore, they may provide an important alternative for warming up activities as they are enjoyable and promote pupils motor development.

\section{Conclusion}

Overall, the present study demonstrated that a short music movement program implemented in the physical education lesson's warm-up phase for 13 weeks can significantly improve fifth and sixth grade elementary school pupils' motor skills related to rhythmic coordination, such as alternating hands drippling and fast sideways stepping. This program could be effectively used to promote motor and psychological development in elementary school complementing regular teaching activities.

\section{References}

Bachmann, M.L. (1991). Dalcroze today: an education through and into music. Oxford University Press: Oxford. Bailey, J. A., \& Penhune, V. B. (2010). Rhythm synchronization performance and auditory working memory in early-and late-trained musicians. Experimental Brain Research, 204(1), 91-101.

Blasi, M. J., \& Foley, M. B. (2006). For parents particularly:"The music, movement, and learning connection": A review. Childhood Education, 82(3), 175-176.

Brown, J., Sherrill, C. \& Gench, B. (1981) 'Effects of an integrated physical education/music program in changing early childhood perceptual-motor performance'. Perceptual and Motor Skills 53(1): 151-4.

Bruininks, R, Bruininks, B. (2006). Bruininks-Oseretsky Test of Motor Proficiency, Second Edition (BOTтм-2). 
Copyright (C) 2006, 2012, 2013 NCS Pearson, Inc. All rights reserved. Retrieved December 2,

2014 from: http://images.pearsonclinical.com/images/Assets/BOT-2/BOT-

2_Complete_Form_Sample_Report.pdf

Bruininks-Oseretsky test of motor proficiency, Review (BOT-2) (2015). Texas Education Agency, TSLAT.

Retrieved April 2, 2019 from http://www.txautism.net/evaluations/the-bruininks-oseretsky-test-of-motorproficiency-second-edition-bot-2

Campbell, D. (2000). The Mozart effect for children. New York, NY: William Morrow. (p.102)

Chatzipandeli, A., Pollatou, E., Diggelidis, N., \& Kourtesis, T. (2007). The Effectiveness of a Music-Movement Program on Manipulative Skills Performance of Six Years Old Children. Inquiries in Sport \& Physical Education 5(1), 19 - 26 published: 27 April 2007.

Cratty, B. (1975). Remedial motor activity for children. London Lea and Febiger 12.

Davidson, M. R. (2004). A phenomenological evaluation: using storytelling as a primary teaching method. Nurse Education in practice, 4(3), 184-189.

Deli, E., Bakle, I., \& Zachopoulou, E. (2006). Implementing intervention movement programs for kindergarten children. Journal of Early Childhood Research, 4(1), 5-18.

Derri, V., Tsapakidou, A., Zachopoulou, E., \& Gini, V. (2001). Complexity of rhythmic ability as measured in preschool children. Perceptual and motor skills, 92(3), 777-785.

Deitz J, Kartin D, Kopp K. (2007). Review of the Bruininks-Oseretsky Test of Motor Proficiency, Second Edition (BOT-2). Physical \& Occupational Therapy In Pediatrics. 27(4):87-102.

Erickson, D. (2004). Creative dance and basketball. Teaching Elementary Physical Education, 15(2), 38- 40.

Faigenbaum, A. D., Bellucci, M., Bernieri, A., Bakker, B., \& Hoorens, K. (2005). Acute effects of different warmup protocols on fitness performance in children. The Journal of Strength \& Conditioning Research, 19(2), 376-381.

Freshwater, A., Sherwood, E., \& Mbugua, E. (2008). Music and physical play: what can we learn from early childhood teachers in Kenya?. Childhood Education, 85(1), 2-5.

Fross, T. (2000). Kehorytmit Emil Jaques-Dalcrozen eurytmian sovelluksena, oppimisprosessina ja opetusmateriaalina [Body percussions as an application, learning process and educational material of Emil Jaques- Dalcrozes eurhythmics]. Pro Gradu JYU.

Gallahue, D. (1996). Developmental Physical Education for Today's Children, 3rd ed. Dubuque, IA:Brown \& Benchmark

Gillespie, C. W., \& Glider, K. R. (2010). Preschool teachers' use of music to scaffold children's learning and behaviour. Early child development and care, 180(6), 799-808.

Garrett, R. (2006). Critical storytelling as a teaching strategy in physical education teacher education. European physical education review, 12(3), 339-360.

Garzotto, F., Paolini, P., \& Sabiescu, A. (2010, June). Interactive storytelling for children. In Proceedings of the 9th International Conference on Interaction Design and Children (pp. 356-359). ACM.

Grahn, J. A., \& Brett, M. (2007). Rhythm and beat perception in motor areas of the brain. Journal of cognitive neuroscience, 19(5), 893-906.

Haapala, E. A., Poikkeus, A-M, Tompuri, T., Kukkonen-Harjula, K., Leppänen P. H. T., Lindi, V., \& Lakka, T. A. (2013). Associations of motor and cardiovascular performance with academic skills in children. Medicine \& Science in Sports \& Exercise, 46, 1016-1024.

Hardman, K., Klein, G., Patriksson, G., Rychtecký, A., \& da Costa, F. C. (2008). Implementation of the Bologna Process and Mod-el Curriculum Development in Physical Education Teacher Education. K. Petry, K. Froberg, A. Madela \& W. Tokarski (2008). Higher Education in Sport in Europe: From Labour Market Demand to Training Supply. Monograph. Maidenhead: Mayer \& Mayer, 56-79.

High, L. (1994). Effects of Selected Rhythmic Teaching Strategies on Beat-Performance Skills of Kindergarten Children. Southeastern Journal of Music Education, 117.

Hotz, A.(1992). Lernen durch Rhythmus. In: H. Rieder (Ed.), Heidelberger Fachgesprache zur Sportwissenschaft. Band, Germany: Heidelberg

Juntunen, M-L., Perkiö, S. \& Simola-Isaksson, I. (2010). Musiikkia Liikkuen [Music with movement]. WSOY

Keetman, G. (1974). Elementaria: first acquaintance with Orff-Schulwerk. Schott \& Co Ltd.

Karageorghis, C. I., \& Terry, P. C. (1997). The psychophysical effects of music in sport and exercise: A review. Journal of Sport Behavior, 20(1), 54.

Kyriazis, D., Pavlidou, E., Barkoukis, V. \& Fotiadou, E. (2017). Effects of a music-movement program in elementary school physical education classes on pupils' rhythmic ability. Journal of Education and Practice $8(35), 17-23$.

Likesas, G., \& Zachopoulou, E. (2006). Music and movement education as a form of motivation in teaching Greek traditional dances. Perceptual and motor skills, 102(2), 552-562.

Lykesas, G., Koutsoumba, M., \& Tyrovola, V. (2009). Creativity as an approach and teaching method of traditional 
Greek dance in secondary schools. Studies in Physical Culture \& Tourism, 16(2).

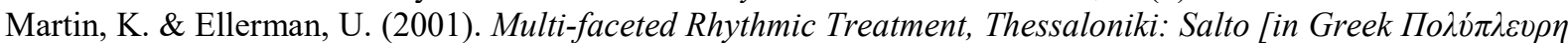

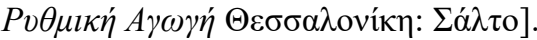

Moore, J. (1984). Rhythm and movement: An objective analysis of their association with music aptitude. Dissertation Abstracts International: 45, $1328 \mathrm{~A}$.

Murray, R. L. (1975). Dance in Elementary Education; A Program for Boys and Girls. Harper and Row Publishers.

Patel, A. D., Iversen, J. R., Chen, Y., \& Repp, B. H. (2005). The influence of metricality and modality on synchronization with a beat. Experimental brain research, 163(2), 226-238.

Pavlidou, E. (1998). Movement and Rhythmic Education in Preschool Education: from theory to practice. [in

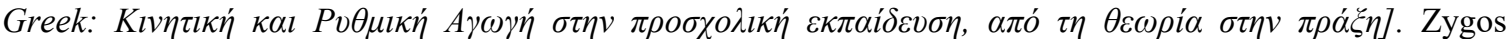
Publications. ISBN: 978-960-8065-87-1, Thessaloniki, 2012, pp. 47-49.

Pavlidou, E., Mertzanidou, O., \& Zissi. V. (2009). Evaluation of Rhythmic Ability in Rhythmic Gymnasts and Non Gymnasts. Inquiries in Sport \& Physical Education 7 (2), 203 - 209.

Pica, R. (1999). Music and the movement program. Teaching Elementary Physical Education, 10(4), 32-33.

Pollatou, E., Pantelakis, G., Agdiniotis, I., Mpenta, N., Zissi, V., \& Karadimou, K. (2012). How is the rhythmic ability of preschool children affected by the implementation of a music movement program? European Psychometricity Journal, 2012; 4(1), 49-56

Salmon, A. (2010). Using music to promote children's thinking and enhance their literacy development. Early child development and care, 180(7), 937-945.

Serbezis, V \& Panagopoulou, V. (2008). Planning and organization of Greek traditional dances Consequence: Teaching program for first-age schoolchildren. Oral \& Poster presentations of the 16th International Congress of Physical Education \& Sport page10. Komotini 16-18 May 2008.

Sousou, S. D. (1997). Effects of melody and lyrics on mood and memory. Perceptual and motor skills, 85(1), 3140.

Tsapakidou, A., Zachopoulou, E., \& Zographou, M. (2001). Early young children's behavior during music and movement program. Journal of Human Movement Studies, 41(5), 333-346.

Venetsanou, F., Donti, O., \& Koutsouba, M. (2014). The effect of a music/movement program on preschooler's motor rhythmic ability. European Psychomotricity Journal, 6(1), 60-73.

Venetsanou, F., \& Kambas, A. (2004). How can a traditional Greek dances programme affect the motor proficiency of pre-school children? Research in Dance Education, 5(2), 127-138.

Weikart, P.S. (1989). Teaching movement and dance. Ypsilanti, Michigan: High/Scope Press

Winsler, A., Ducenne, L., \& Koury, A. (2011). Singing one's way to self-regulation: The role of early music and movement curricula and private speech. Early Education and Development, 22(2), 274-304.

Zachopoulou, E., Derri, V., Chatzopoulos, D., \& Ellinoudis, T. (2003). Application of Orff and Dalcroze activities in preschool children: Do they affect the level of rhythmic ability?. Physical Educator, 60(2), 50.

Zachopoulou, E., Tsapakidou, A., \& Derri, V. (2004). The effects of a developmentally appropriate music and movement program on motor performance. Early Childhood Research Quarterly, 19(4), 631-642. 